\title{
Meta
}

Journal des traducteurs

Translators' Journal

\section{LOMBEZ, CHRISTINE, dir. (2012) : Traduire en langue française \\ en 1830. Arras : Artois Presses Université, 208 p.}

\section{Brigitte Lépinette}

Volume 59, numéro 1, avril 2014

URI : https://id.erudit.org/iderudit/1026480ar

DOI : https://doi.org/10.7202/1026480ar

Aller au sommaire du numéro

Éditeur(s)

Les Presses de l’Université de Montréal

ISSN

0026-0452 (imprimé)

1492-1421 (numérique)

Découvrir la revue

Citer ce compte rendu

Lépinette, B. (2014). Compte rendu de [Lombez, Christine, dir. (2012) :

Traduire en langue française en 1830. Arras : Artois Presses Université, 208 p.]

Meta, 59(1), 217-223. https://doi.org/10.7202/1026480ar

Ce document est protégé par la loi sur le droit d'auteur. L'utilisation des services d’Érudit (y compris la reproduction) est assujettie à sa politique d'utilisation que vous pouvez consulter en ligne.

https://apropos.erudit.org/fr/usagers/politique-dutilisation/
Cet article est diffusé et préservé par Érudit.

Érudit est un consortium interuniversitaire sans but lucratif composé de l’Université de Montréal, l’Université Laval et l’Université du Québec à Montréal. Il a pour mission la promotion et la valorisation de la recherche. https://www.erudit.org/fr/ 
training to interpreting evaluation, which covers all related branches of Chinese interpreting and presents "the features of the Chinese interpreting scene" (p. 2). The contributors from Hong Kong, Taiwan and the mainland constructed a whole map of Chinese interpreting and its research, and found a way in this book to express the latest research results, which have already deflected speculative discussion and practitioners' personal narratives about practice and training in the early stage of translation studies in China. The eagerness of the Chinese interpreting community to make contact and express itself is apparent in reading between the lines of these papers. While readers are not fully apprised of the past and present efforts of Chinese interpreters and academic researchers, this book will surely prove valuable since it opens a window for those outside to glimpse the present status of interpreting practice and research in China, and for those "Chinese interpreters" inside to contact the outside world and show their potential. Thus it is a stimulating and inspiring book.

Chen Zhijie

Nanjing University of Information Science and Technology; Nanjing, Chine

\section{REFERENCES}

House, Juliane (2001): Translation Quality Assessment: Linguistic Description versus Social Evaluation. Meta: Translators' Journal. 46(2): 243-257.

Newmark, Peter (1981): Approaches to Translation. London: Pergamon Press.

Lombez, Christine, dir. (2012): Traduire en langue française en 1830. Arras: Artois Presses Université, 208 p.

Ce volume comprend 13 textes qui ont d'abord fait l'objet d'une présentation orale lors du colloque «Traduire en langue française en 1830 » tenu à Nantes les 13, 14 et 15 novembre 2008 ${ }^{1}$. Malgré cette origine, qui suppose toujours une plus grande variété thématique et méthodologique que dans un volume dont les parties ont initialement été conçues comme complémentaires, présentant donc a priori une cohérence forte, l'ensemble ici décrit a néanmoins une unité, assurée en premier lieu par une délimitation chronologique très précise (1830) et, ensuite, par le fait que la langue d'arrivée des textes pris en compte est, dans tous les cas, le français. Comme nous le verrons, les objets d'étude sélectionnés y sont néanmoins variés presse, roman, etc. mais aussi réflexions autour du phénomène de la traduction dite historique ou encore contextualisation sous divers points de vue (culturel, littéraire, etc.), de traductions, etc. -, tout comme l'est le traitement qu'on leur assure. Pourtant, les experts en traductologie historique, mais aussi les historiens et les spécialistes en littérature et en littérature comparée y trouveront amplement leur compte, comme nous essaierons de le montrer.

Dans un texte liminaire à caractère introductoire, Yves Chevrel retrace à grands traits le panorama de la production littéraire autour de 1830, montrant que les traductions sont loin d'en être absentes, même si dans l'histoire littéraire française elles n'y apparaissent d'ordinaire que rarement. Il explique ainsi le fait:

[1]es travaux sur l'histoire de la littérature française sont [...] tributaires d'un choix méthodologique persistant qui [leur fait mettre] l'accent sur les créations et, de ce fait, négliger la réalité des offres de lecture ou de représentation théâtrale. (p. 11)

Cependant, Y. Chevrel insiste, à juste titre, sur le fait que l'histoire du livre actuelle, et, entre autres, l'étude bibliométrique très documentée de Martyn Lyons (Lyons 1987), fournit un tableau éloquent des lectures des Français (et de celles qui sont traduites de l'anglais, en particulier). Il montre aussi la lente, mais incessante pénétration des littératures étrangères en France: les Français sont loin de ne lire alors que des créations françaises, comme la presse de l'époque permet de le constater. L'année 1830, prise comme référence centrale de l'ensemble des études, est évidemment explicable d'un simple point de vue historique, mais, comme l'indique l'auteur de l'introduction du volume, elle marque le début du «changement des mentalités qui est en train de s'opérer en France à l'égard des œuvres traduites» (p. 19). Ainsi est justifiée dans l'étude traductologique cette référence à l'année 1830, qui voit: «de nouvelles impulsions, de nouvelles questions, suscitées par un appel de l'étranger, de plus en plus entendu - et rendu plus accessible par les traductions» (p. 20).

Dans l'étude intitulée "Traductions de textes étrangers dans les périodiques français en $1830 »$ (p. 21-34), Patrick Berthier s'intéressera aux périodiques français dont Chevrel avait montré, quelques pages plus haut, l'importance croissante. La littérature n'est pas le genre le mieux représenté dans ces traductions. La manière de traduire des journalistes est aussi examinée, et l'auteur souligne la fréquence de l'attitude qu'il qualifie (de façon euphémistique) d'«interventionniste». Ainsi, par exemple, Le correspondant (mars 1831) offre à ses lecteurs une lettre à propos de laquelle il n'hésite pas à préciser :

Nous donnons [transcrivons pour les lecteurs du journal] la lettre telle qu'elle est dans l'original, seulement comme elle est fort longue, 
nous avons cru devoir supprimer quelques répétitions et des détails qui ne sont pas d'un intérêt général. (p. 30)

De même, ainsi que le souligne l'auteur de l'article (mais sans en offrir une explication), les traductions anonymes ne sont pas rares dans cette presse de 1830. En tout cas, ce qui se dégage de cette étude de Berthier, c'est l'ampleur du champ de la traduction dans la presse qu'il y découvre, bien que, pour lui, les incursions qui y ont été faites jusqu'à maintenant par les chercheurs sont encore trop peu nombreuses pour que l'on puisse en tirer des conclusions définitives. Pourtant, c'est à très juste titre qu'il conclut: «Si partiel que soit l'examen proposé ici, il permet déjà de confirmer, d'un point de vue général, à quel point la presse est un "répercuteur" d'influences et d'entrées étrangères » (p. 34).

Lieven d'Hulst présente au lecteur une réflexion mettant en préambule une constatation qui nous semble personnellement essentielle en histoire de la traduction. La traduction, activité interdisciplinaire - a fortiori l'histoire de la traduction -, l'est moins peut-être par les données que des disciplines connexes peuvent lui apporter que parce que ces dernières

procurent [...] un appareil conceptuel et méthodologique précieux qui complète sur des points essentiels les démarches traductologiques à caractère herméneutique: la prise en compte de tous les agents impliqués dans la production et dans la distribution des traductions, l'analyse des contraintes politiques et économiques qui pèsent sur les échanges culturels, les logiques nationales et internationales du marché du livre, etc. (p. 36)

La question de la bibliométrie par les historiens et l'articulation des données que celle-ci permet dans ces œuvres historiques avec l'outillage habituel est malheureusement, selon l'auteur, encore «hésitante» (p. 36). Pourtant, bien qu'avec toutes les précautions que suscite cette situation, c'est aux données bibliographiques concernant les œuvres traduites en France en 1830 que Lieven d'Hulst s'attachera dans le volume que nous présentons ici. Sa source principale - dont il envisagera avec le plus grand soin les caractéristiques ainsi que les conséquences de ces dernières pour son analyse - sera La bibliographie de France, registre créé par Napoléon en 1810. Sous le titre Palmarès des disciplines (p. 40), l'auteur de l'étude classe thématiquement les traductions et peut ainsi faire constater que les traductions de BellesLettres, en 1830, occupent encore la première place, reléguant les traductions des sciences et des arts à un second rang pour une dizaine d'années encore.
Les langues-sources sont celles des pays voisins (anglais, allemand, italien, grec - moderne?- et, enfin, espagnol). La place encore significative du latin nous rappelle l'importance qu'assume toujours cette langue chez les lettrés du XIX ${ }^{\mathrm{e}}$ siècle. Au palmarès des auteurs traduits, Walter Scott figure en première place; en effet, 1830 représente: «une année phare pour les traductions de l'auteur écossais qui domine de loin le paysage de l'édition des traductions [...] couvr[ant] plus de $11 \%$ de l'ensemble des 440 titres " traduits en France cette année-là (p. 40). Il serait intéressant de comparer cette statistique avec celle d'autres pays pour y calibrer l'importance du phénomène «Scott» à la même époque ${ }^{2}$. Il conviendra de souligner une donnée apportée par Lieven d'Hulst - le texte (1830) d'Auguste Dufauconpret (1767-1844), le traducteur français le plus prolifique (grâce aux œuvres de James Fenimore Cooper et, surtout, de Walter Scott) - sera utilisé par le premier traducteur espagnol de Scott en 1836. Il s'agira donc d'une traduction indirecte, cas assez fréquent en Espagne à l'époque que nous envisageons. Inutile de souligner l'intérêt, dans ce cas et dans nombre d'autres, qu'il y a à mieux connaître la réception européenne d'œuvres qui dessinèrent une véritable trame internationale et configurèrent largement le goût de l'époque. Dans son enquête, Lieven d'Hulst établit aussi une typologie des «modes de présentation des traductions»: traduction "nouvelle", "littérale", "imitée", "libre", la "traduction nouvelle" étant la plus souvent présente. L'auteur s'interroge, sans opter pour une réponse définitive sur cette "nouveauté" qui pourrait être, selon nous, surtout redevable au souci de l'éditeur de favoriser des ventes. Dans le même domaine en rapport direct avec l'histoire du livre, nous pouvons lire la liste des éditeurs français, liste qui demanderait qu'on s'y arrête un peu plus longuement, et ce, sous la houlette de spécialistes de l'histoire de l'édition du livre français (dans ses aspects économiques, culturels, idéologiques, etc.), discipline à laquelle appartient aussi l'histoire de la traduction. Cette histoire de l'édition expliquerait aussi pourquoi, dans la partie finale de l'étude d'Hulst, par exemple, la province est peu représentée sous la rubrique "Lieux d'édition".

En somme, un bel exemple d'étude, celui d'Hulst, qui a montré tout l'intérêt d'envisager la traduction comme un objet historique, partie intégrante d'une culture réceptrice dans ses divers aspects (idéologiques, économiques, littéraires, etc.), plus que comme un texte dont seuls les sens seraient à interroger. Le sujet du colloque - la détermination de l'année 1830 - et la visée que d'Hulst a adoptée dans la plupart de ses précédentes études le conduisaient naturellement à ce genre d'analyse, mais inutile de dire qu'on y 
reconnaît l'ampleur de vues de ce maître en histoire de la traduction.

L'éditrice a placé, après le texte de d'Hulst, deux analyses concernant, la première, une traduction du russe - d'Ivan Khemnister, un fabuliste dont l'œuvre sera traduite en vers les plus exacts possible (p. 58) - et la seconde, de l'allemand - l'historien Berthold Georg Niebuhr, le traducteur étant, dans ce cas, Marie Philippe Aimé de Golbéry ${ }^{3}$ - grosso modo, la démarche est la même dans ces deux études, respectivement, de Françoise Genevray et d'Agnès Graceffa: elles apportent toutes deux des données sur les auteurs et sur les traducteurs, sur la traduction elle-même, sur les raisons pour lesquelles cette dernière a été entreprise et, enfin, sur sa réception.

La première étude (de Genevray) contribue à dessiner la trame des échanges entre la lointaine Russie et la France, éloignement social, moral, politique, etc. qui rend la traduction difficile. Avec la seconde analyse (d'Agnès Graceffa), dans une traduction également problématique, l'on découvre un pan de l'apport méthodologique de l'Allemagne à la science historique en France:

la Römische Geschichte illustre une conception nouvelle de la science historique, elle valide le principe novateur d'une histoire scientifique qui s'efforce de comprendre une société passée à travers ses propres structures de pensée. Cette démarche annonce la posture de l'historicisme tel qu'il sera bientôt défini par L. von Ranke (1795-1886). (p. 66)

L'auteure de la contribution commente longuement, et avec très grande pertinence, cette influence de Niebuhr sur l'historiographie européenne, dans ce qui nous semble des pages hautement intéressantes. Tout aussi intéressantes sont celles consacrées au dilemme auquel fut confronté le traducteur du XIX ${ }^{e}$ siècle: devait-il écrire pour la science ou pour la littérature? Dans le cas de Golbéry, le style sera sacrifié «à l'impératif scientifique", fait qui déterminera in fine la préférence des lecteurs français pour une histoire vraiment française. Les pages d'Agnès Graceffa prennent en compte avec une grande érudition les paramètres de la question historique en France autour de 1830 et réussissent, de ce fait, à éclairer la place qu'un texte traduit y occupe, ainsi que le rejet pour des raisons, disons «nationalistes " pour faire court mais il y en a d'autres plus consistantes -, de l'œuvre de Niebuhr. L'auteure rappellera significativement une citation d'Alfred Blot (1866: ix) : «l'Allemagne n'a fait que nous renvoyer nos propres découvertes enveloppées et obscurcies par les nuages d'une érudition pédantesque». Si l'Angleterre et l'Allemagne n'oublieront pas complètement Niebuhr et Golbéry, en France et en langue française, ces derniers perdront tout crédit. Finalement, l'intérêt tout particulier que revêt cette étude est manifeste pour qui s'intéresse à la traduction du XIX ${ }^{\mathrm{e}}$ siècle, mais aussi simplement à l'historiographie de ce siècle, qui est réaffirmée par une très complète bibliographie dénotant l'ample connaissance du sujet de la part de l'auteure.

Le texte de Rainier Grutman se situe à la croisée des chemins de l'histoire de la littérature et de celle de la traduction, s'amorçant à partir d'une polémique musclée entre Jules Janin et Désiré Nisard en 1834. L'un des deux personnages, Nisard, avait déclaré sans ambages: "La traduction ne mène plus à rien» et représenterait: "une sorte d'abdication ou d'abnégation du genre [littéraire] et de ceux qui le pratiquent» (p. 78). L'auteur de l'étude découvre ce qui, selon Nisard, serait la cause de cette décadence: les retraductions d'œuvres classiques qui dépossèdent le traducteur de tout prestige littéraire. Les positions de Janin et de Nisard, relativement anecdotiques en ellesmêmes, s'inscrivent en réalité dans une polémique plus vaste: «entre l'esthétique romantique qui célèbre la création originale au détriment des modèles et l'esthétique (néo)classique, qui réactive ces derniers par divers moyens dont la traduction " (p. 79). Plusieurs pages intéressantes analysent le statut du traducteur et de son activité - peu reconnue socialement - à cette époque, grâce à la relecture, entre autres, de la préface aux Études françaises et étrangères d'Emile Deschamps, ici considérée comme une véritable défense et illustration de la traduction. Rainier Grutman cite à l'appui d'une thèse selon laquelle le traducteur n'a pas un rôle si négligeable qu'il ne l'a été dit - le rôle de François-Adolphe Loève-Veimar, traducteur des Contes d'Hoffmann qui servit de «filtre» à ses lecteurs français - filtre considéré de deux points de vue, celui du choix du texte à traduire et de la manière dont le traducteur décide de le traduire, la traduction allant bien au-delà du simple transvasement linguistique. Après une longue discussion sur la manière de traduire de Loève-Veimar et sur la réception - parfois extrêmement défavorable de ses traductions, Grutman insiste sur la place non insignifiante que croit néanmoins occuper ce traducteur dans la République des Lettres (l'étude souligne d'ailleurs que cette place lui sera refusée avec parfois une extrême dureté, par des écrivains comme Balzac, entre autres). Ce chapitre étudiant une polémique à propos du statut littéraire de la traduction et de celui, plutôt social, mais aussi littéraire, du traducteur prouverait, en dernier ressort, avec le cas particulier ici présenté, que le rôle du traducteur est en train de se transformer et de se cloisonner par rapport à l'œuvre de l'écrivain. Cette évolution laissera finalement le travail du traducteur comme une simple «annexe» de l'œuvre 
de l'écrivain, en un bilan dont on se demande ce qu'il vaut aujourd'hui, concernant les traductions et traducteurs actuels...

L'étude de Claudine Le Blanc décrit d'abord le contexte historique dans lequel naît l'intérêt pour la langue sanskrite en Europe et, en particulier, en France, où exista une chaire de sanskrit dès 1814 (la première à être créée en Europe). Pourtant, dès la fin du XviII ${ }^{\mathrm{e}}$ siècle, les traductions de cette langue furent le fait des Anglais. C'est pourquoi Le Blanc s'attachera d'abord à situer dans son contexte historique, intellectuel et linguistique la première traduction anglaise de William Jones (1796-1794), un orientaliste qui «découvrit le théâtre indien et la pièce que la tradition tenait pour la plus belle: Abhijñânashakuntalâ» qu'il traduisit (p. 95). L'auteure de l'étude nous rappelle aussi que, côté français, l'intérêt pour le sanskrit - nous l'avons vu avec la création de la chaire de cette langue - est présent. Ainsi, le célèbre orientaliste Sylvestre de Sacy enseignait le persan à Paris, où il eut pour élève un personnage qui intéressera ici au premier chef: Antoine-Léonard de Chézy (17741832). Titulaire à partir de 1814 de la chaire de sanskrit du collège royal de France, Chézy traduisit La reconnaissance de Sacountala, drame sanscrit [...] (Paris, Librairie orientale Dondey-Dupré). Comme le documente Le Blanc, cet érudit jouera un rôle important dans la diffusion, auprès d'un public éclairé, de la littérature sanskrite. Dans une exposition historique précise, cette auteure décrit la retraduction et traduction à la fois de Shakuntalâ par Chézy qui y corrige Jones, s'opposant à lui dans la position qu'il soutient concernant les traductions orientales (p. 99). En effet, l'Anglais était persuadé que: «la traduction de textes appartenant à une culture aussi étrangère que la civilisation de l'Inde classique se confronte de façon démultipliée à un impossible» (p. 99). Au contraire, le Français s'efforcera de souligner la valeur universelle de la pièce sanskrite, pensant qu'elle saura toucher «le lecteur digne d'apprécier ce drame» (p. 100). Par ailleurs, l'auteure fait noter que la traduction de Chézy, dont elle souligne l'option d'hellénisation du drame indien, ne visera pas seulement la diffusion d'un type de littérature exotique, mais elle sera accompagnée de tout un appareil critique - le fait ne surprenant pas de la part du professeur du collège royal de France. Annexes et notes en nombre considérable constituent un véritable travail scientifique sur l'œuvre originale, même si de nombreux commentaires attachés au texte de Chezy - dont Le Blanc fournit et analyse des exemples (p. 102-104) viennent critiquer la traduction de Jones. Le travail du Français, «homme à l'érudition aventureuse, ombrageuse et frémissante» selon la description de l'auteure (p. 108), sera finalement préféré par cette dernière pour les qualités stylistiques (en rapport avec sa personnalité, l'on suppose) de sa traduction. Pour ce qui est de la réception et du rôle dans les lettres françaises de Shakuntalâ, le bilan serait plutôt négatif, selon l'auteure de l'étude. La traduction ne s'est pas intégrée dans la culture occidentale: «[E]lle a touché les gens de lettres [à son époque]. Elle a échoué, en revanche, à instituer durablement, à l'instar de la littérature grecque, la littérature sanskrite» (p. 107). La version de Chézy, finalement, relèverait plutôt du domaine de l'histoire de la traduction.

Sur Madame de Rochmondet, traductrice révolutionnaire, Benoît Léger n'apporte que peu de données précises, et ce, pour la bonne raison qu'il n'y en a pas beaucoup. Même son appartenance au genre féminin pourrait être mise en question (si Rochmondet était un pseudonyme, ce qui constitue un mystère). D’abord, l'auteur de l'étude décrit l'évolution de la manière de traduire dominante au début du XIX ${ }^{\mathrm{e}}$ siècle, qui commence à mettre en doute le bien-fondé de la traduction des Belles Infidèles. Pour apporter de l'eau à ce moulin, Léger cite par exemple:

le polémiste Eugène Aroux [dans sa traduction du Paradise lost] dénonce la version paraphrasante à laquelle se livrait Delille qui [aurait] péché trop souvent par l'infidélité et l'inexactitude. (p. 11)

Par ailleurs, entre 1815 et 1830, il se serait produit une professionnalisation de la traduction (p. 113), et madame de Rochmondet y aurait participé - bien qu'avec une certaine ambigüité ou un manque de définition selon l'auteur - et aurait alors défendu, dans ses Études sur la traduction de l'anglais, or, Lessons on the French translation (de Rochmondet et Léger 2009), une position révolutionnaire (?) en faveur d'une éthique professionnelle de la traduction. L'étude s'achève sur une affirmation qui peut ne pas faire l'unanimité des lecteurs: «La voix [de Rochmondet, préfacière, critique et traductrice] est omniprésente [dans ses études] mais cette voix reste, malheureusement, anonyme» (p. 115). La biographie d'un traducteur expliquerait-elle toujours ses positions traductologiques?

Christine Lombez analyse une anthologie intitulée Poésies européennes de Léon Halévy, éditée pour la première fois en 1827 et rééditée en 1830. L'objectif de l'auteure de l'étude est de s'interroger sur la composition même de l'anthologie ainsi que sur les stratégies de traduction du compilateur: "partagé entre "imitation" et "traduction" " (p. 119). Classiquement, l'étude s'ouvre sur une description de l'ouvrage, en particulier, sur le contenu de sa préface, puis décrit le parcours biographique de Léon Halévy: "polygraphe, historien, auteur dramatique, poète très actif », bref, un personnage influent de son temps (p. 121). À juste titre, Lombez 
considère qu'une anthologie, dans le choix de ses éléments, est directement reliée à la culture et au goût, ainsi qu'à l'idéologie et même à la pédagogie d'une époque. La liste détaillée que fournit l'étude de Lombez sur les pièces intégrées est intéressante dans la mesure où la langue grecque moderne y est spécialement bien représentée (bien mieux que le russe ou l'espagnol, par exemple). Le fait a conduit l'auteure à regarder de plus près dans cet ensemble néohellénique que sélectionna Léon Halévy et il lui a permis de voir qu'il s'agit, assez curieusement, de l'utilisation d'un canevas en prose - il n'y aura donc pas traduction à proprement parler - et, dans un second temps, d'une mise en vers; dans ce cas, il faudra parler d'imitation, ou encore de réécriture. Le procédé s'inscrirait donc sous le titre générique d'adaptation, que nous aurions presque du mal à appeler traductologique. Le terme d'imitation qu'emploie l'auteure de l'étude nous cantonnerait dans le domaine strictement littéraire.

La première traduction française de Kleist - Michael Kohlhoas le marchand de chevaux et autres contes - consiste surtout en une "observation critique» de cette traduction faite par les Cherbuliez, éditeurs-libraires genevois en 1830 (p. 159). La conclusion de Jean-Yves Masson, auteur de l'étude de cette traduction, qui a voulu «juger» le Kleist français en mettant en contraste le texte allemand, la traduction des Genevois et une autre traduction française, est assez pessimiste, même s'il avait initialement opté, pour une (selon ses propres mots) neutralité bienveillante:

Force est de constater [dans la traduction des Cherbuliez] que nous avons été conduits à constituer une liste de défauts, pour certains fort graves et que nous n'avons que bien peu de qualités à reconnaître [...]. Les traducteurs [...] ont détruit une grande partie de ce qui fait l'art [du] conteur, le caractère envoûtant du continuum narratif ainsi que nombre d'allusions qui permettent d'en saisir l'arrière-plan métaphysique. (p. 159)

Un tel constat permet de comprendre, ainsi qu'en fait état l'étude de Masson, qu'une mauvaise traduction - celle qui vide un texte de la substance de son écriture - n'assume pas son rôle d'intermédiaire littéraire entre deux nations.

Gabriel-Louis Moyal interroge - évidemment, autour de 1830 - dans ce cas, les contextes français et anglais dans lesquels «s'imaginent et se destinent les traductions» (p. 164). S’appuyant sur Toury (1995), Moyal postule, comme ce dernier, l'interdépendance des différentes méthodes, des fonctions et des approches en traductologie, ce qui l'amène à replacer chaque traduction

dans un système intriqué de relations sociales, politiques et économiques, littéraires, etc. et comme contribuant en même temps à l'évolution et modification de ces systèmes, à leur adaptation à un environnement autre, provoqué et instauré aussi par le fait même de cette traduction. (p. 165)

C'est dans cette optique que sont examinées les biographies contextualisées et les traductions, d'une part, de Helen Maria Williams (Relation des événements qui se sont passés en France depuis le débarquement de Napoléon Buonaparte [...] suivie d'observations sur l'état présent de la France et sur l'opinion publique, Paris: Dentu, 1816), Anglaise qui a vécu en France depuis la Révolution et, d'autre part, La France en 1829 et 1830 (Paris: H. Fournier, 1830) de Lady Morgan. Cet examen permet à Moyal la présentation d'une réflexion historique, sociologique, politique et idéologique sur les rapports entre la France et l'Angleterre vers 1830 .

Le chapitre extrêmement bien documenté de María del Rosario Álvarez Rubio (encore enrichi de nombreuses notes de bas de page, parfois même, démesurément longues, à notre sens) nous situe cette fois dans le domaine espagnol et signale trois traductions éditées à Paris en 1830: Histoire de la monja alférez, Aben Humeya ou la révolte des Maures sous Philippe II, drame historique, Les Romances du Cid [...], suivi de l'abrégé historique de la vie du Cid; par le Chevalier Regnard [...]. Álvarez Rubio s'étend d'abord avec pertinence sur la dénomination de traducteur, qui, comme ce fut le cas pour le Chevalier Regnard, fut appliquée avant 1830 à divers officiers qui avaient participé à la guerre dans la Péninsule sous Napoléon. L'auteure considère alors les poèmes originaux du Cid, qui avaient fasciné Regnard, bien que celui-ci ne fût pas «un spécialiste de cette culture méridionale» (p. 180). Elle rappelle que cet intérêt a pour contexte littéraire "les théories romantiques de Schlegel $[. .$.$] » et «s'inscrit dans une révision de la$ littérature espagnole, de son identité historique et de son appartenance à la modernité européenne» dont sont responsables les Ilustrados (p. 181). Ce renouveau d'intérêt pour le Cid coïncide aussi, hors d'Espagne, avec «l'influence herdienne qui identifiait le littéraire au primitif et qui se manifeste alors dans le goût pour les ballades et les légendes » (p. 181). C'est ainsi que la traduction de Regnard constitue un maillon de la longue chaîne que sera la diffusion du Romancero et, en particulier, celle du Cid. Dans ces pages qui retracent avec une grande érudition la généalogie compliquée (laquelle remonte au XviII ${ }^{\mathrm{e}}$ siècle) des traductions du Cid en France, l'auteure examine le travail de Regnard, dont la principale source serait Les romances du Cid (Paris: Delaunay, 1814) de Creuzé de Lesser, et elle compare cette traduction de 1830 avec la précédente de 1814 . La traduction de 
1830 constitue «une démarche plus respectueuse» (bien qu'il s'agisse pourtant d'une traduction dite libre par Regnard) que celle de Creuzé de Lesser (p. 186). Finalement, Álvarez Rubio insistera sur le fait que Regnard fera appel pour élaborer sa traduction, aux «modèles français d'élocution contemporains des romances, surtout à Froissart qui lui est familier» (p. 187), car il se propose «de transmettre la simplicité et l'énergie stylistique des octosyllabes originaux", ne prétendant pas "à la recherche esthétique de sa source française » (p. 187). Il faut saluer que, dans cette étude riche autant que dense, soit présent, avec un appareil critique considérable, le contexte littéraire et culturel de 1830, que connaît si bien l'auteure et qui, finalement, au-delà des options personnelles des traducteurs, permet de mieux comprendre les options stylistiques de différentes traductions d'un original à des époques différentes.

Dans l'étude intitulée "L'ardente querelle autour des œuvres de Tacite en 1830 ", Frédéric Weinmann analyse le travail "homologue" de Louis Panckouke et de Jean-Louis Burnouf (17751844). Les deux hommes aux profils intellectuels et sociaux tout à fait différents traduisirent les Euvres de C.C. Tacite (1830). Le travail de Panckouke, dont l'auteur de l'article décrit avec précision la gestation, expose des options qui vont à l'encontre de celles qui furent prises avant lui, comme le montre la préface de ce texte, véritable manifeste contre la traduction classique et contre l'Université. D'autre part, les options de Burnouf sont, pour l'auteur de l'article ici pris en compte, à la fois: «floues et d'apparence moderne [mais il s'y] cache une conception en réalité ancienne» (p. 193) qui va être mise en évidence de manière précise dans divers textes en contraste (p. 195, 196, 197) mettant face à face les deux traducteurs contemporains. L'auteur analyse avec beaucoup de finesse les deux théories antagoniques de la traduction. Ainsi, résumant sa doctrine classique, Burnouf affirme "qu'il faut conserver sous les costumes français la noblesse et la majesté de la toge romaine» (p. 199). Au contraire, la citation de Panckouke montre que ce dernier s'est obligé à une traduction littérale

qui a soin, non seulement de pas intervertir l'ordre des pensées, car c'est toujours détruire leur gradation, mais en respectant même l'ordre des mots, car agir autrement c'est détruire leurs images; [...] J'ai porté le scrupule jusqu'à ne pas changer la place d'une épithète». (p. 198)

Cette traduction littérale était dans l'air du temps, pouvant être héritière de la traduction interlinéaire du XVIII ${ }^{\mathrm{e}}$ siècle: «d'après les principes de MM. De Port-Royal, Dumarsais, Beauzée et des plus grands maîtres» (p. 198).
Le parcours dans ces 13 textes dont l'unité était a priori marquée par une donnée chronologique externe - la date de 1830 - et le fait - interne - que les traductions envisagées aient pour langue d'arrivée le français, a donné lieu à un ensemble d'une richesse remarquable en même temps que d'une diversité radicale, aussi bien du point de vue des textes eux-mêmes que de celui du contexte intellectuel et littéraire qui les a vu naître et des traducteurs dont les options divergent dans la plupart des cas. Pourtant, cette diversité est, dans une perspective scientifique, hautement positive: tous ces textes attestent, avec une fidélité totale, de l'ampleur actuelle du "territoire" dont, à bon droit, s'approprie actuellement l'histoire de la traduction, activité qui jouxte, de façon de plus en plus évidente, nombre de disciplines, par là, connexes ${ }^{4}$, auxquelles elle emprunte des objets mais aussi des méthodes. Il est difficile pour une spécialiste en histoire de la traduction, comme celle qui écrit ces lignes, de ne pas se réjouir de la publication de ce volume et de ne pas saluer le travail de chaque auteur et, en même temps, celui de l'éditrice - qui a suscité ces études et les a réunies-, comme une grande réussite dans le domaine de la «traductologie historique».

BRIGITTE LÉPINETTE
Valencia, Universitat de València-IULMA,
Espagne

\section{NOTES}

1. Comme l'indique l'Avant-propos, ces actes sont «publiés dans le cadre du projet HTLF (Histoire des traductions en langue française), sous la direction d'Yves Chervel et de JeanYves Masson».

2. Voir, par exemple, au sujet de l'influence de Walter Scott en Espagne, les classiques: Peers 1926, Zellers 1931 ou, plus récemment, García Gonzalez 2005 et, tout spécialement, García Gonzalez 2009. Dans cette dernière étude figure la liste complète des traductions de Walter Scott en espagnol au XIX ${ }^{e}$ siècle. Voir Walter Scott (Dernière mise à jour: 7 janvier 2013): Consulté le 23 avril 2014, <http://www. walterscott.lib.ed.ac.uk/etexts/novels.html\# waverley>; et Bibliothèque nationale de France (BNF). Gallica - Bibliothèque numérique. Euvres de Walter Scott. Tome 3: Consulté le 23 avril 2014, <http://gallica.bnf.fr/ark:/12148/ bpt6k283244>.

3. Golbéry est un spécialiste de l'Antiquité, comme le met en évidence la bibliographie de l'auteure.

4. Voir Lieven d'Hulst, dans ce même volume (p. 34-48).

\section{RÉFÉRENCES}

BLOT, Alfred (1866): Introduction. In: Louis DE BeAufort. Dissertation sur les cinq premiers 
siècles de l'histoire romaine. Nouvelle édition avec une introduction et des notes par Alfred BLот. Paris: Maillet, iii-xiv.

García Gonzalez, José Enrique (2005): Consideraciones sobre la influencia de Walter Scott en la novela histórica española del siglo XIX. CAUCE. 28:109-119.

García Gonzalez, José Enrique (2009): Estudio y edición traductológica digital de Waverley, de Walter Scott, en traducción anónima, Barcelona, Librería-Imprenta de Oliva, 1836. In: Carmen Acuña Partal et Marcos RodríGUEZ Espinosa, dir. Archivo y edición digital de textos literarios y ensayísticos traducidos al español y tratados sobre traducción del siglo XIX. Granada: Editorial Atrio, 1-15.

Lyons, Martyn (1987): Le triomphe du livre. Une histoire sociologique de la lecture dans la France $d u X I X^{e}$ siècle. Paris: Promodis.

Peers, Edgar Allison (1926): Studies in the Influence of Sir Walter Scott in Spain. Revue Hispanique. 68:1-160.

DE Rochmondet, madame G.M. et LÉGer, Benoît (2009): Études sur la traduction de l'anglais, or, Lessons on the French translation. Introduction, notes et bibliographie de Benoît LÉGER. Ottawa: Presses de l'Université d'Ottawa.

Toury, Gideon (1995): Descriptive Translation Studies - And Beyond. Amsterdam/Philadelphie: John Benjamins.

Zellers, Guillermo (1931): Influencia de Walter Scott en España. Revista de Filología Española. 18:149-162.

Hamilton, Grant et Lavallée, François (2012): Tweets et gazouillis: pour des traductions qui chantent. Montréal: Linguatech éditeur inc., $163 \mathrm{p}$.

À l'heure où les Kim Thúy, Bernard Pivot et Alexandre Jardin se lancent dans la Twitterature et écrivent des romans ou des nouvelles (allez savoir) de 140 caractères, voilà que deux réviseurs québécois publient un recueil de conseils aux traducteurs sous la même forme condensée. Grant Hamilton et François Lavallée sont deux figures bien connues de la traduction à Québec, au Québec et au-delà de ses frontières, deux réviseurs respectés à juste titre. Le recueil qu'ils nous proposent renferme de nombreuses pistes de solutions des plus intéressantes.

C'est la maison d'édition Linguatech qui s'est occupée de la livrée: typographie impeccable, mise en page soignée, jaquette élégante, couverture rehaussée d'une reproduction d'une œuvre de Robert Dubuc. Un bel objet en somme, qui allie l'utile à l'agréable. En quatrième de couverture, l'acheteur potentiel est informé que l'ouvrage comprend un index de 3000 entrées, soit en gros 40 pages sur les 160 que comporte le recueil. C'était en somme le prix à payer pour passer de deux outils virtuels à indexation intégrée, des fils Twitter, à un mode de présentation plus traditionnel sans perte de terrain sur le plan de la convivialité.

Indépendamment de la qualité de l'ouvrage, on pourrait remettre en question la pertinence de sa publication même sous format papier. Si, comme l'a déclaré le co-fondateur de l'Institut comparé de twittérature, Jacques Fréchette, avec Twitter, «chaque chair de poule devient prétexte d'une expression ${ }^{1} »$, est-il pertinent d'en publier un recueil?

Loin de constituer de l'acharnement pédagogique, cette transition témoigne plutôt selon moi d'un désir candide de faire profiter le plus grand nombre de judicieux conseils de réviseurs hors pair, comme l'exprime François Lavallée, ainsi que d'une ouverture vers les médias modernes de Linguatech. La démarche de Grant Hamilton était encore plus directe: c'est la réponse à la question «en quoi Twitter peut-il aider les traducteurs?» qui a mené à la publication de l'ouvrage.

D’emblée, les deux auteurs reconnaissent la contrainte souvent décriée du nombre de caractères tout en soulignant les conséquences heureuses de cette restriction: limpidité de l'expression, facilité de mémorisation et court-circuitage des argumentations. Il leur arrive toutefois de contourner cette difficulté en consacrant plusieurs gazouillis au même terme: Grant Hamilton décortique le terme rassembleur en 6 fois 140 caractères (tweets 482 à 487) alors qu'il en consacre 4 au terme mandat (361 à 364). François Lavallée, souvent porté à faire travailler son lecteur par ailleurs, abuse moins de ces séries d'instantanés sans mise en contexte. À la réflexion, avec ces séries de gazouillis consacrés à un même terme, on n'est pas très loin d'un court article dans un ouvrage plus traditionnel du type Le traducteur averti $i^{2}$ ou Les trucs d'anglais qu'on a oublié de vous enseigner ${ }^{3}$. À l'instar de la bande dessinée, où une série de gags en une case d'André Franquin, par exemple, tout en permettant d'aborder sous divers angles les mêmes facettes de son personnage Gaston Lagaffe, n'a pas la même saveur qu'une bande dessinée en une planche, ces séries de tweets demeurent des images autonomes; il y manque le «récit», que le lecteur doit trouver par lui-même.

Les deux auteurs adoptant à l'occasion des points de vue différents, il peut être utile d'examiner séparément leur démarche. Les tweets de Grant Hamilton présentent souvent l'avantage d'être très pratiques, et il a pris grand soin de les classer en 76 catégories précises si l'on excepte l'inévitable fourre-tout du type General Advice. Voici une entrée typique: «342 Conférence: A FR conférence is a talk or lecture in EN, whereas an EN "conference" is usually a congrès in FR». Sur 